\title{
An Impedance Boundary Condition EFIE that is Low-Frequency and Refinement Stable
}

\author{
Alexandre Dély, Student Member, IEEE, Francesco P. Andriulli, Senior Member, IEEE, and Kristof Cools.
}

\begin{abstract}
A discretization of the IBC-EFIE is introduced that (i) yields the correct solution at arbitrarily small frequencies, (ii) requires for its solution a number of matrix vector products bounded as the frequency tends to zero and as the mesh density increases. The low frequency stabilization is based on a projectorbased discrete Helmholtz splitting, rescaling, and recombination that depends on the low frequency behavior of both the EFIE operator and the surface impedance condition. The dense mesh stabilization is a modification of the perfect electric conductor operator preconditioning approach taking into account the effect on the singular value spectrum of the IBC term.
\end{abstract}

Index Terms-Scattering, Impedance Boundary Conditions, Preconditioning, Low Frequency, Boundary Element Method.

\section{INTRODUCTION}

$\mathbf{T}$ HE scattering of time-harmonic electromagnetic waves by non-penetrable objects can be efficiently modeled by the Electric Field Integral Equation (PEC-EFIE) [1]. In this equation, the unknown is the tangential trace of the magnetic field on the boundary of the scatterer. For perfectly conducting objects this trace equals the current induced at the surface of the scatterer and the EFIE is equivalent to the statement that the tangential components of the scattered field generated by this induced current negate the incident field at the surface of the scatterer.

The EFIE can be generalized to model scattering by a much wider class of non-penetrable objects. This is classically obtained by leveraging impedance boundary conditions (IBCs) [2]. These conditions, which will be the starting point for this paper, enforce a relationship between between tangential components of electric and magnetic fields, i.e. $\boldsymbol{e}_{t}=z \hat{\boldsymbol{n}} \times \boldsymbol{h}_{t}$, or equivalently $\boldsymbol{m}=-z \hat{\boldsymbol{n}} \times \boldsymbol{j}$, where $(\boldsymbol{j}, \boldsymbol{m})$ are the equivalent electric and magnetic surface currents, and $z$ is a so-called surface impedance. When the IBC condition is combined with the representation formulas, it is possible to obtain a first kind equation which is usually known in literature as the Impedance Boundary Condition EFIE (IBC-EFIE) [3]. This equation includes an extra term that takes into account the surface impedance condition. This technique is widespread and is known to provide accurate models of scattering in a wide variety of scenarios. This notwithstanding several recent contributions have sensibly advanced the original integral equation approach by proposing combined field formulations [4], discretizations based on dual elements [5], self-dual schemes [6], and generalized impedance boundary conditions [7], [8].

This work was supported in part by the French DGA agency in the framework of grants for doctoral theses in cooperation between France and United Kingdom.
It is well known that the standard EFIE operator suffers from ill-conditioning when the frequency is low or the discretization density is high. This is often referred to as low-frequency and dense-mesh breakdown, respectively (see [9] and references therein). Although the remarkable advancement in the topic, all IBC formulations currently available are plagued by at least one of the two breakdowns. The concurrent solution of both breakdowns for the IBC-EFIE will be the aim of this paper.

Recently, by leveraging quasi-Helmholtz projector decomposition techniques, the standard EFIE has been rendered numerically stable and accurate at very low frequencies approaching and including zero [10]. On one hand this allows the EFIE to be used in multi-scale problems, without the necessity to couple the EFIE with a dedicated eddy current modeling tool on the boundary of the low and extremely low frequency regions. On the other hand, the EFIE system can be preconditioned using Calderon preconditioning techniques, resulting in linear systems whose condition numbers are virtually independent of the mesh size. This again is a prerequisite for the modeling of scattering by multi-scale problems and in addition allows for the recovery of the exact solution up to arbitrary accuracy.

These methods unfortunately do not trivially carry over to the IBC-EFIE. The reason is that the presence of the term stemming from the IBC affects the scaling of the system matrix blocks in a Helmholtz decomposed basis and the asymptotic behavior of the singular values as the mesh parameter tends to zero. In this contribution, a new formulation of the IBC-EFIE is introduced that (i) yields the correct solution at arbitrarily small frequencies, (ii) has a condition number bounded as the frequency tends to zero, and (iii) has a condition number bounded as the mesh parameter goes to zero. The low frequency stabilization is based on a discrete Helmholtz splitting, rescaling, and recombination that depends on the low frequency behavior of both the EFIE operator and the surface impedance condition. The dense grid stabilization is a modification of the PEC Calderon approach taking into account the effect on the singular value spectrum of the IBC term.

This paper is organized as follow: in Section II the notation is set and the IBC-EFIE is constructed based on the representation theorem. The discretization of the IBC-EFIE leveraging both primal and dual finite element spaces is revisited. In Section III the low frequency behavior of the Helmholtz components of the solution of the IBC-EFIE is studied by considering its solution on a spherical surface in terms of vector spherical harmonics. From this analysis rescaling operators are constructed resulting in a system that 
is well conditioned up to arbitrarily low frequencies and that is not susceptible to numerical cancellation in the presence of limited machine precision or quadrature errors. In Section $\mathrm{IV}$, the condition number at fixed frequency as the mesh parameter tends to zero is studied. As in Section III, the analysis is first performed for the solution of the IBC on spherical surfaces and then generalized by considering the behavior of the system matrix blocks in a discrete Helmholtz decomposed basis. Based on the conclusions of this analysis, a Calderon-like approach is proposed an the details of its construction elucidated. Finally in Section VI numerical results are presented demonstrating the claims made in this introduction. Both benchmark examples and real life scenarios are considered.

\section{NOTATION AND BACKGROUND}

Consider a domain $\Omega$ with boundary $\Gamma$. The domain is embedded in a medium characterized by a permittivity $\epsilon$ and permeability $\mu$, corresponding to an impedance $\eta=\frac{\mu \omega}{k}$ and wave number $k=\omega \sqrt{\epsilon \mu}$. On $\Gamma$ the exterior normal is denoted $\hat{\boldsymbol{n}}$.

The incident fields are $\boldsymbol{e}^{i}$ and $\boldsymbol{h}^{i} .(\boldsymbol{e}, \boldsymbol{h})$ are the solutions to Maxwell's equations in the exterior domain $\mathbb{R}^{3} \backslash \Omega$ which satisfy Sommerfeld's radiation conditions. The representation theorem (for the exterior domain) allows to express the incident fields in terms of electric and magnetic equivalent currents $\boldsymbol{j}$ and $\boldsymbol{m}$ as

$$
\left(\begin{array}{cc}
\frac{1}{2}+K & \eta T \\
-\frac{1}{\eta} T & \frac{1}{2}+K
\end{array}\right)\left(\begin{array}{c}
\boldsymbol{m} \\
\boldsymbol{j}
\end{array}\right)=\left(\begin{array}{c}
\boldsymbol{e}^{i} \times \hat{\boldsymbol{n}} \\
\hat{\boldsymbol{n}} \times \boldsymbol{h}^{i}
\end{array}\right)
$$

with $\boldsymbol{j}=\hat{\boldsymbol{n}} \times \boldsymbol{h}$ and $\boldsymbol{m}=\boldsymbol{e} \times \hat{\boldsymbol{n}}$, and where

$$
\begin{aligned}
T(\boldsymbol{j}) & =i k T_{s}(\boldsymbol{j})+\frac{1}{i k} T_{h}(\boldsymbol{j}) \\
& =-i k \hat{\boldsymbol{n}} \times \int_{\Gamma} \frac{e^{-i k R}}{4 \pi R} \boldsymbol{j}\left(\boldsymbol{r}^{\prime}\right) d \boldsymbol{r}^{\prime} \\
& +\frac{1}{i k} \hat{\boldsymbol{n}} \times \nabla \int_{\Gamma} \frac{e^{-i k R}}{4 \pi R} \nabla^{\prime} \cdot \boldsymbol{j}\left(\boldsymbol{r}^{\prime}\right) d \boldsymbol{r}^{\prime}
\end{aligned}
$$

and

$$
K(\boldsymbol{j})=-\hat{\boldsymbol{n}} \times \text { p.v. } \int_{\Gamma} \nabla \frac{e^{-i k R}}{4 \pi R} \times \boldsymbol{j}\left(\boldsymbol{r}^{\prime}\right) d \boldsymbol{r}^{\prime},
$$

are the single and double layer boundary integral operators for the Maxwell system. Here, $R=\left|\boldsymbol{r}-\boldsymbol{r}^{\prime}\right|$ and p.v. denotes the Cauchy principal value of the integral.

In order to be solved, the representation formulas for the exterior domain in (1) must be complemented by either the formulas for the interior domain (this is what leads to integral equations for penetrable objects) or with a boundary condition. This contribution focuses on the latter case and we enforce the following relationship between $\boldsymbol{j}$ and $\boldsymbol{m}$

$$
\boldsymbol{m}=-z \hat{\boldsymbol{n}} \times \boldsymbol{j},
$$

with $z$ scalar (complex). Equation (5) is classically known as impedance boundary condition.

Using (5) in the first equation of (1) results in the Impedance Boundary Condition EFIE (IBC-EFIE)

$$
S(\boldsymbol{j})=\eta T \boldsymbol{j}-\left(\frac{1}{2}+K\right) z(\hat{\boldsymbol{n}} \times \boldsymbol{j})=\boldsymbol{e}^{i} \times \hat{\boldsymbol{n}}
$$

\section{A. Discretization strategy}

The discretization strategy proposed in [4] is briefly summarized here to fix the notation. In order to discretize (6), the surface $\Gamma$ is approximated by a flat faceted triangular mesh $\mathcal{T}$ comprising $V$ vertices, $N$ edges and $F$ faces. On this mesh the basis of Rao-Wilton-Glisson (RWG) functions [11], $\boldsymbol{f}_{m}$, $m=1, \ldots, N$, is constructed to discretize the current $\boldsymbol{j}$. The RWG basis functions are normalized such that the integral of their normal component over the defining edge equals one (this differs from a factor edge length from the definition found in [11]). The Buffa-Christiansen (BC) functions introduced in [12], $\boldsymbol{g}_{m}, m=1, \ldots, N$ are used to discretize the current $\boldsymbol{m}$. These functions are linear combinations of RWG basis functions defined on the barycentric refinement of $\mathcal{T}$.

Substituting the approximations $\boldsymbol{j} \approx \sum_{n=1}^{N} \mathrm{j}_{n} \boldsymbol{f}_{n}$ and $\boldsymbol{m} \approx$ $\sum_{n=1}^{N} \mathrm{~m}_{n} \boldsymbol{g}_{n}$ and testing (5) with $\left(\boldsymbol{f}_{m}\right)_{m=1}^{N}$ gives [4]

$$
\mathrm{m}=-z \mathbf{G}_{m i x}^{-1} \mathbf{G j}
$$

where $\left(\mathbf{G}_{m i x}\right)_{i, j}=\left\langle\hat{\boldsymbol{n}} \times \boldsymbol{f}_{i}, \boldsymbol{g}_{j}\right\rangle,(\mathbf{G})_{i, j}=\left\langle\boldsymbol{f}_{i}, \boldsymbol{f}_{j}\right\rangle$ and $\langle.,$. denotes the $L^{2}(\Gamma)$ inner product. Then the equation (6) is discretized as

$$
\mathbf{S} \mathbf{j}=\left(\eta \mathbf{T}-z\left(\mathbf{K}+\frac{1}{2} \mathbf{G}_{m i x}\right) \mathbf{G}_{m i x}^{-1} \mathbf{G}\right) \mathbf{j}=\mathrm{V}
$$

where $(\mathbf{S})_{i, j}=\left\langle\hat{\boldsymbol{n}} \times \boldsymbol{f}_{i}, S \boldsymbol{f}_{j}\right\rangle,(\mathbf{T})_{i, j}=\left\langle\hat{\boldsymbol{n}} \times \boldsymbol{f}_{i}, T \boldsymbol{f}_{j}\right\rangle$, $(\mathbf{K})_{i, j}=\left\langle\hat{\boldsymbol{n}} \times \boldsymbol{f}_{i}, K \boldsymbol{g}_{j}\right\rangle$ and $(\mathrm{V})_{m}=\left\langle\hat{\boldsymbol{n}} \times \boldsymbol{f}_{m}, \boldsymbol{e}^{i} \times \hat{\boldsymbol{n}}\right\rangle$.

The coefficients of both RWG and BC functions allow for a discrete Helmholtz decomposition [13]. First, define the connectivity matrices $\boldsymbol{\Lambda} \in \mathbb{R}^{N \times V}$ and $\boldsymbol{\Sigma} \in \mathbb{R}^{N \times F}$

$$
\begin{array}{ll}
\boldsymbol{\Lambda}_{m i}= \pm 1 & \text { if edge } m \text { leaves/arrives at } \\
& \begin{array}{l}
\text { vertex } i, 0 \text { otherwise } \\
\boldsymbol{\Sigma}_{m j}= \pm 1
\end{array} \\
& \text { if edge } m \text { is on the boundary of face } \\
j \text { clockwise/countercw, } 0 \text { otherwise }
\end{array}
$$

The space of RWG coefficients $\left\{\mathrm{j} \in \mathbb{C}^{N}\right\}$ is now split into the direct sum of two subspaces. The subspace $\operatorname{Im} \boldsymbol{\Sigma}$ of RWG stars, which for convenience will also be denoted $\Sigma$, and its $l^{2}(N)$ orthogonal complement $\Lambda H$ (note that for simply connected surfaces $\Lambda H=\Lambda=\operatorname{Im} \boldsymbol{\Lambda}$. Here, $l^{2}(N)$ is $\mathbb{C}^{N}$ endowed with the Euclidean inner product.

The condition number of equation (8) grows when the frequency decreases or the discretization density increases. These effects are inherited from the low-frequency breakdown $(k \rightarrow 0)$ and the dense mesh breakdown $(h \rightarrow 0)$ of the standard EFIE operator. This paper focuses on the solution of these breakdowns for the IBC-EFIE. It is worth mentioning that the IBC-EFIE at high frequency is subject to spurious resonances, as well as the regularized version presented here. A resonant-free formulation could be obtained from the new equation we will propose here by combining it with a magnetic counterpart in a CFIE fashion following, for example, a strategy similar to the one in [4]. This will be a topic for future research. 


\section{ANALYSIS AND REgUlARIZATION OF THE} LOW-FREQUENCY BREAKDOWN OF IBC-EFIE

To gain initial insight in the low-frequency behavior of the IBC-EFIE, a Mie series analysis based on an expansion in vector spherical harmonics is presented in this section.

Although this strategy does not strictly apply to the general case, its findings could be generalized to arbitrary smooth geometries following similar arguments as in [14].

Define the vector spherical harmonics

$$
\begin{aligned}
& \boldsymbol{X}_{l m}(\theta, \varphi)=\frac{a}{i \sqrt{l(l+1)}} \hat{\boldsymbol{n}} \times \nabla Y_{l m}(\theta, \varphi) \\
& \boldsymbol{U}_{l m}(\theta, \varphi)=\hat{\boldsymbol{n}} \times \boldsymbol{X}_{l m}(\theta, \varphi)
\end{aligned}
$$

with $Y_{l m}$ the scalar spherical harmonics and $a$ the sphere radius. The vector spherical harmonics are singular vectors of both $T$ and $K$ operators. In particular, it holds [14] that

$$
\begin{aligned}
S\left(\boldsymbol{X}_{l m}\right) & =\left[\left(-\eta H_{l}(k a)-z i H_{l}^{\prime}(k a)\right) J_{l}(k a)\right] \boldsymbol{U}_{l m} \\
S\left(\boldsymbol{U}_{l m}\right) & =\left[\left(\eta H_{l}^{\prime}(k a)-z i H_{l}(k a)\right) J_{l}^{\prime}(k a)\right] \boldsymbol{X}_{l m}
\end{aligned}
$$

where $H_{l}, J_{l}, H_{l}^{\prime}$ and $J_{l}^{\prime}$ denote the Riccati-Hankel (of second kind) and Bessel functions and their derivatives, respectively.

Also, by noting $S^{*}$ the adjoint operator of $S$, the eigenvalues of $S^{*} S$ (that are the squares of the singular values of $S$ ) are given by the following equations

$$
\begin{aligned}
S^{*} S\left(\boldsymbol{X}_{l m}\right) & =\left(\left|-\eta H_{l}(k a)-z i H_{l}^{\prime}(k a)\right| J_{l}(k a)\right)^{2} \boldsymbol{X}_{l m} \\
S^{*} S\left(\boldsymbol{U}_{l m}\right) & =\left(\left|\eta H_{l}^{\prime}(k a)-z i H_{l}(k a)\right| J_{l}^{\prime}(k a)\right)^{2} \boldsymbol{U}_{l m} .
\end{aligned}
$$

The asymptotic behavior of these special functions for $k \rightarrow 0$ (see, for example, [15]), with the assumption that $z=o\left(k^{-1}\right)$ when $k \rightarrow 0$, which holds in all cases of practical interest, results in the following asymptotic estimates,

$$
\begin{aligned}
S\left(\boldsymbol{X}_{l m}\right) & =\left[\underset{k \rightarrow 0}{O}\left(\frac{i \eta k a}{l}+z\right)\right] \boldsymbol{U}_{l m} \\
S\left(\boldsymbol{U}_{l m}\right) & =\left[\underset{k \rightarrow 0}{O}\left(\frac{i \eta l}{k a}\right)\right] \boldsymbol{X}_{l m} \\
S^{*} S\left(\boldsymbol{X}_{l m}\right) & =\left[\underset{k \rightarrow 0}{O}\left(\left|\frac{i \eta k a}{l}+z\right|^{2}\right)\right] \boldsymbol{X}_{l m} \\
S^{*} S\left(\boldsymbol{U}_{l m}\right) & =\left[\underset{k \rightarrow 0}{O}\left(\left(\frac{\eta l}{k a}\right)^{2}\right)\right] \boldsymbol{U}_{l m} .
\end{aligned}
$$

By fixing the maximum number of terms $l_{\max }$ in the Mie series expansion, the scaling of the condition number of $\mathbf{S}$ in (8) can be computed. The maximum singular value of $\mathbf{S}$ is read from (20) and its minimum singular value from (19). From this it follows that the condition number of $\mathbf{S}$ scales as

$$
\operatorname{cond}(\mathbf{S})=\underset{k \rightarrow 0}{O}\left(\frac{\eta l_{\max }}{k a\left|\frac{i \eta k a}{l_{\max }}+z\right|}\right) \text {. }
$$

This results in a low-frequency breakdown every time $z=$ $o\left(k^{-1}\right)$. It should also be noted that when $z=0$ we recover the quadratic-in-frequency growth of the condition number which characterizes the classical EFIE for metallic surfaces.

This section is concerned with the low frequency behavior of the condition number so global constant factors and the truncation point $l_{\max }$ of the Mie series will be omitted in the following computations. Stating these asymptotic expressions allows to generalize the conclusions in this section to arbitrary geometries [14].

To reflect this generalization, for a general structure we identify $a=D / 2$, with $D$ the diameter of the geometry under consideration. Moreover, in a vector harmonics expansion truncated at $l_{\max }$ the number of degrees of freedom is $O\left(l_{\max }^{2}\right)$. Comparing this to the number of degrees of freedom in a boundary element method discretization $O\left(D^{2} / h^{2}\right)$ leads to the approximate relationship $l_{\max } \approx D / h$.

In addition to conditioning problems, low-frequency regimes are often associated to numerical cancellations in the solution current. This is the case for the standard EFIE [10]. In the following we will assess the problem for IBC-EFIE in the case of plane wave incidence. For a plane wave the following scalings for the right-hand-side hold [10]

$$
\begin{aligned}
\left\langle\boldsymbol{X}_{l m}, \hat{n} \times \boldsymbol{e}^{i}\right\rangle & =\underset{k \rightarrow 0}{O}(1) \\
\left\langle\boldsymbol{U}_{l m}, \hat{n} \times \boldsymbol{e}^{i}\right\rangle & =\underset{k \rightarrow 0}{O}(i k a),
\end{aligned}
$$

where $\langle.,$.$\rangle is the L^{2}(\Gamma)$ inner product. Dividing the scaling of the right hand side coefficients by the corresponding ones of the left hand side (respectively (23) with (17), and (22) with (18)) results in the following scalings for the electric current

$$
\begin{aligned}
& \left\langle\boldsymbol{X}_{l m}, \boldsymbol{j}\right\rangle=\underset{k \rightarrow 0}{O}\left(\frac{i k a}{i \eta k a / l+z}\right) \\
& \left\langle\boldsymbol{U}_{l m}, \boldsymbol{j}\right\rangle=\underset{k \rightarrow 0}{O}\left(\frac{i k a}{l \eta}\right) .
\end{aligned}
$$

Then, using the impedance boundary condition (5) results in these scalings for the magnetic current

$$
\begin{aligned}
& \left\langle\boldsymbol{X}_{l m}, \boldsymbol{m}\right\rangle=\underset{k \rightarrow 0}{O}\left(\frac{z i k a}{\eta l}\right) \\
& \left\langle\boldsymbol{U}_{l m}, \boldsymbol{m}\right\rangle=\underset{k \rightarrow 0}{O}\left(\frac{z i k a}{i \eta k a / l+z}\right) .
\end{aligned}
$$

The analysis above shows that, differently from the standard EFIE, for the IBC-EFIE numerical cancellations do not always occur. Indeed, when $k \rightarrow 0$ the solenoidal and non solenoidal parts of the current scale as $O(1)$ and $O(k)$ for the EFIE $(z=0)$ which effectively results in a numerical cancellation. For the IBC-EFIE, both parts of the current have the same frequency scaling $O(k)$ if $z \neq 0$ when $k \rightarrow 0$ : no numerical cancellation will occur at low frequency.

\section{A. Solution of the low-frequency problems of the IBC-EFIE}

This subsection will show how to solve the low-frequency problems occurring in the solution of the IBC-EFIE. Our strategy will be based on the quasi-Helmholtz projectors introduced in [10] for the standard EFIE. We briefly define these projectors here for the sake of completeness. Given an RWG coefficient vector $\mathrm{j} \in \mathbb{C}^{N}$ it holds that

$$
\mathrm{j}=\mathbf{P}^{\Sigma} \mathbf{j}+\mathbf{P}^{\Lambda H} \mathbf{j},
$$

with $\mathbf{P}^{\Sigma}=\boldsymbol{\Sigma}\left(\boldsymbol{\Sigma}^{T} \boldsymbol{\Sigma}\right)^{+} \boldsymbol{\Sigma}^{T}$ and $\mathbf{P}^{\Lambda H}=\mathbf{I}-\mathbf{P}^{\Sigma}$. Note that the action of the pseudo inverse on any array can be computed 
in linear complexity using off-the-shelf algorithms [13]. The splitting is orthogonal in the space of coefficients $l^{2}(N)$. To minimise the effect of the frequency scaling on the growth of the condition number, we will rescale the projectors such that the low frequency behaviour of the corresponding eigenvalues will be the same. The previous analysis suggests the following definitions

$$
\begin{aligned}
& \mathbf{M}_{1}=\mathbf{P}^{\Sigma}+\frac{1}{i k a} \mathbf{P}^{\Lambda H} \\
& \mathbf{M}_{2}=i k a \mathbf{P}^{\Sigma}+\frac{i \eta k a}{i \eta k a+z} \mathbf{P}^{\Lambda H} .
\end{aligned}
$$

The following low-frequency regularization for the IBCEFIE is proposed:

$$
\mathrm{M}_{1} \mathrm{SM}_{2} \mathrm{Y}=\mathrm{M}_{1} \mathrm{~V}
$$

where, as before, $\mathbf{S}=\eta \mathbf{T}-z\left(\mathbf{K}+\frac{1}{2} \mathbf{G}_{m i x}\right) \mathbf{G}_{m i x}^{-1} \mathbf{G}$ and where, $\mathrm{j}$ is then retrieved as

$$
\mathrm{j}=\mathrm{M}_{2} \mathrm{Y} .
$$

The analysis based on spherical harmonics described in the previous section suggests that (31) is indeed immune from low-frequency problems. As mentioned in the derivation, the length scale $a$ is proportional to the diameter $D$ of the structure under consideration. As the asymptotic behavior does not depend upon its exact value, it will be put to 1 meter in the numerical experiments in Section VI.

Applying $\mathbf{M}_{1}$ and $\mathbf{M}_{2}$ on the equation (8) results in a system that is immune to the low frequency breakdown. This can be seen in the following way. Define

$$
\begin{aligned}
\mathbf{T} & =i k \mathbf{T}_{s}+\frac{1}{i k} \mathbf{T}_{h}, \\
\mathbf{K}^{+} & =\left(\mathbf{K}+\frac{1}{2} \mathbf{G}_{m i x}\right) \mathbf{G}_{m i x}^{-1} \mathbf{G},
\end{aligned}
$$

then the low frequency behavior of the frequency regularized IBC-EFIE is

$$
\begin{aligned}
\mathbf{M}_{1} \mathbf{S M}_{2} & =\mathbf{P}^{\Sigma}\left(-k^{2} a \eta \mathbf{T}_{s}+a \eta \mathbf{T}_{h}-i k a z \mathbf{K}^{+}\right) \mathbf{P}^{\Sigma} \\
& +\mathbf{P}^{\Sigma}\left(-\frac{k^{2} a \eta^{2}}{i \eta k a+z} \mathbf{T}_{s}-\frac{i k a \eta z}{i \eta k a+z} \mathbf{K}^{+}\right) \mathbf{P}^{\Lambda H} \\
& +\mathbf{P}^{\Lambda H}\left(i k \eta \mathbf{T}_{s}-z \mathbf{K}^{+}\right) \mathbf{P}^{\Sigma} \\
& +\mathbf{P}^{\Lambda H}\left(\frac{i k \eta^{2}}{i \eta k a+z} \mathbf{T}_{s}-\frac{\eta z}{i \eta k a+z} \mathbf{K}^{+}\right) \mathbf{P}^{\Lambda H} \\
& =\mathbf{P}^{\Lambda H}\left(\frac{i k \eta^{2}}{i \eta k a+z} \mathbf{T}_{s}-\frac{\eta z}{i \eta k a+z} \mathbf{K}^{+}\right) \mathbf{P}^{\Lambda H} \\
& +a \eta \mathbf{P}^{\Sigma} \mathbf{T}_{h} \mathbf{P}^{\Sigma}-z \mathbf{P}^{\Lambda H} \mathbf{K}^{+} \mathbf{P}^{\Sigma}+\underset{k \rightarrow 0}{O}(k)
\end{aligned}
$$

It can be read off from this asymptotic estimate that the residual condition number only depends on the geometry and the asymptotic value of $z(\omega)$ : this condition number will be independent of $k$ when $\lim _{k \rightarrow 0} z(\omega)$ is finite. This is the case for a wide range of physically and technically relevant impedance models including, for example, the Drude model. The condition number, and in turn the number of iterations required to solve the discrete IBC-EFIE does not depend on $k$. Otherwise said, (31) is immune from low-frequency breakdown.

\section{Analysis and Regularization of the Dense MESH BREAKDOWN OF IBC-EFIE}

As in the case of the low-frequency breakdown, spherical harmonics can be used to analyze the conditioning problem of the IBC-EFIE when the discretization density increases (i.e., $h \rightarrow 0$ ). In fact, equation (13) and (14), together with the asymptotic scalings of spherical harmonics and their derivatives for high order provide

$$
\begin{aligned}
S\left(\boldsymbol{U}_{l m}\right) & =\left[\underset{l \rightarrow \infty}{O}\left(\frac{i \eta l}{k a}\right)\right] \boldsymbol{X}_{l m} \\
S\left(\boldsymbol{X}_{l m}\right) & =\left[\underset{l \rightarrow \infty}{O}\left(\frac{i \eta k a}{l}+z\right)\right] \boldsymbol{U}_{l m} .
\end{aligned}
$$

As we have said before, in a vector harmonics expansion truncated at $l_{\max }$ the approximate relationship $l_{\max } \approx a / h$ holds and thus the estimate for the condition number of the boundary element system in terms of the mesh parameter $h$

$$
\operatorname{cond}(\mathbf{S})=\underset{h \rightarrow 0}{O}\left(\frac{\eta}{k h|i \eta k h+z|}\right)=\underset{h \rightarrow 0}{O}\left(\frac{1}{h}\right)
$$

where the last passage is obtained under the hypothesis that $z \neq 0$. It should be noted that differently from the standard EFIE, that shows a conditioning which is $O\left(1 / h^{2}\right)$, the IBCEFIE condition number is only linearly growing with the inverse of the mesh parameter $h$. This is so because the branch of the spectrum (see (38)) associated to the (compact) operator $T_{s}$ is dominated in the IBC-EFIE by the presence of the identity which is absent in the standard EFIE.

\section{A. Solution of the dense mesh problems of the IBC-EFIE}

A further addition to the new formulation in (31) will result in an equation that is immune from both low frequency and dense mesh breakdown. Consider the dual projectors [10] $\mathbf{P}^{\Lambda}=\boldsymbol{\Lambda}\left(\boldsymbol{\Lambda}^{T} \boldsymbol{\Lambda}\right)^{+} \boldsymbol{\Lambda}^{T}$ and $\mathbf{P}^{\Sigma H}=\mathbf{I}-\mathbf{P}^{\Lambda}$. Also, define the matrix $\mathbb{T}_{s}$ as the operator $T_{s}$ discretized with the BC basis function: $\left(\mathbb{T}_{s}\right)_{i, j}=\left\langle\hat{\boldsymbol{n}} \times \boldsymbol{g}_{i}, T_{s} \boldsymbol{g}_{j}\right\rangle$ Define also the following rescaling operator

$$
\mathbf{M}_{3}=\frac{1}{a} \mathbf{P}^{\Sigma H} \mathbb{T}_{s} \mathbf{P}^{\Sigma H}+\mathbf{P}^{\Lambda} .
$$

This rescaling operator is designed to provide a regularization with the operator $T_{s}$ only where needed, while the part of the spectrum which is already regular (due to the presence of the identity in $S$, see considerations after (39)) is not further regularized. Finally, the regularized low-frequency and dense grid stable IBC-EFIE we propose reads

$$
\mathbf{M}_{3} \mathbf{G}_{m i x}^{-1} \mathbf{M}_{1} \mathbf{S M}_{2} \mathbf{Y}=\mathbf{M}_{3} \mathbf{G}_{m i x}^{-1} \mathbf{M}_{1} \bigvee
$$

The reader should notice that the inverse of the mix-Gram matrix $\mathbf{G}_{m i x}^{-1}$ is used to link the RWG and BC basis functions. 


\section{B. Properties of the formulation}

The low frequency properties of the formulation in (41) are unchanged by the additional presence of the rescaling operator $\mathbf{M}_{3}$, so that the analysis in subsection III-A applies here unaltered. Regarding the dense grid behavior of the equation, the rationale behind equation (41) can be further understood by using spherical harmonics. In fact the continuous counterpart $M_{3}$ of the matrix $\mathbf{M}_{3}$ has the following spherical harmonics mappings

$$
\begin{aligned}
M_{3}\left(\boldsymbol{U}_{l m}\right) & =\frac{1}{a} T_{s}\left(\boldsymbol{U}_{l m}\right)=\left[\underset{l \rightarrow \infty}{O}\left(\frac{1}{l}\right)\right] \boldsymbol{X}_{l m} \\
M_{3}\left(\boldsymbol{X}_{l m}\right) & =\boldsymbol{U}_{l m} .
\end{aligned}
$$

The above mappings, combined with (37) and (38), results in a conditioning for (41) which is independent on both frequency and $h$.

\section{IMPLEMENTATION RELATED DETAILS}

At extremely low frequency, some precautions must be taken to avoid numerical cancellation in the computation of (41) right hand side. Especially when computing $V$ for $a$ plane wave $e^{i}=e_{0} e^{i k \hat{r} \cdot r^{i}}$, the solenoidal part $\mathrm{V}_{e x t}$ should be computed using the extracted form $e^{i k \hat{\boldsymbol{r}} \cdot r^{i}}-1$ so that $\left(\mathrm{V}_{e x t}\right)_{m}=\left\langle\hat{\boldsymbol{n}} \times \boldsymbol{f}_{m},-\hat{\boldsymbol{n}} \times \boldsymbol{e}_{0}\left(e^{i k \hat{\boldsymbol{r}} \cdot r^{i}}-1\right)\right\rangle$ because at high frequency $\mathbf{P}^{\Lambda H} \mathrm{~V}_{\text {ext }}=\mathbf{P}^{\Lambda H} \mathrm{~V}$, but at low frequency only $\mathbf{P}^{\Lambda H} \bigvee_{\text {ext }}$ provides the accurate result

$$
\begin{aligned}
\mathrm{V}_{1} & =\frac{1}{i k a} \mathbf{M}_{3} \mathbf{G}_{m i x}^{-1} \mathbf{P}^{\Lambda H} \mathrm{~V}_{e x t} \\
\mathrm{~V}_{2} & =\mathbf{M}_{3} \mathbf{G}_{m i x}^{-1} \mathbf{P}^{\Sigma} \mathrm{V} .
\end{aligned}
$$

This results from the explicit development of the operator $\mathbf{M}_{1}$ (see (29)) to have $\mathbf{M}_{3} \mathbf{G}_{m i x}^{-1} \mathbf{M}_{1} \mathrm{~V}=\mathrm{V}_{1}+\mathrm{V}_{2}$.

Also, to compute the system matrix in (41) and avoid numerical cancellation at extremely low frequency, the properties $\mathbf{T}_{h} \mathbf{P}^{\Lambda H}=\mathbf{P}^{\Lambda H} \mathbf{T}_{h}=0$ and $\mathbf{P}^{\Sigma} \mathbf{T}_{h} \mathbf{P}^{\Sigma}=\mathbf{T}_{h}$ [10] must be explicitly enforced, i.e. by defining

$$
\begin{aligned}
& \mathbf{A}_{1}=-\mathbf{M}_{3} \mathbf{G}_{m i x}^{-1} \mathbf{M}_{1} z\left(\mathbf{K}+\frac{\mathbf{G}_{m i x}}{2}\right) \mathbf{G}_{m i x}^{-1} \mathbf{G M}_{2} \\
& \mathbf{A}_{2}=\eta i k \mathbf{M}_{3} \mathbf{G}_{m i x}^{-1} \mathbf{M}_{1} \mathbf{T}_{s} \mathbf{M}_{2} \\
& \mathbf{A}_{3}=a \eta \mathbf{M}_{3} \mathbf{G}_{m i x}^{-1} \mathbf{T}_{h}
\end{aligned}
$$

we have $\mathbf{M}_{3} \mathbf{G}_{\text {mix }}^{-1} \mathbf{M}_{1} \mathbf{S} \mathbf{M}_{2}=\mathbf{A}_{1}+\mathbf{A}_{2}+\mathbf{A}_{3}$, thus (41) becomes

$$
\left(\mathbf{A}_{1}+\mathbf{A}_{2}+\mathbf{A}_{3}\right) \mathrm{Y}=\mathrm{V}_{1}+\mathrm{V}_{2} \text {. }
$$

The system (49) is the one that is actually solved. To get the electric current $\mathrm{j}$ from $\mathrm{Y}$ we use (32) and to get the magnetic current $m$ we use the IBC (7). So, the solenoidal part and non solenoidal part of the currents are retrieved with

$$
\begin{aligned}
\mathrm{j}_{s} & =\frac{i \eta k a}{i \eta k a+z} \mathbf{P}^{\Lambda H} \mathbf{Y} \\
\mathrm{j}_{n s} & =i k a \mathbf{P}^{\Sigma} \mathbf{Y} \\
\mathrm{m}_{s} & =-z \mathbf{G}_{m i x}^{-1} \mathbf{G j}_{n s} \\
\mathrm{~m}_{n s} & =-z \mathbf{G}_{m i x}^{-1} \mathbf{G j}_{s} .
\end{aligned}
$$

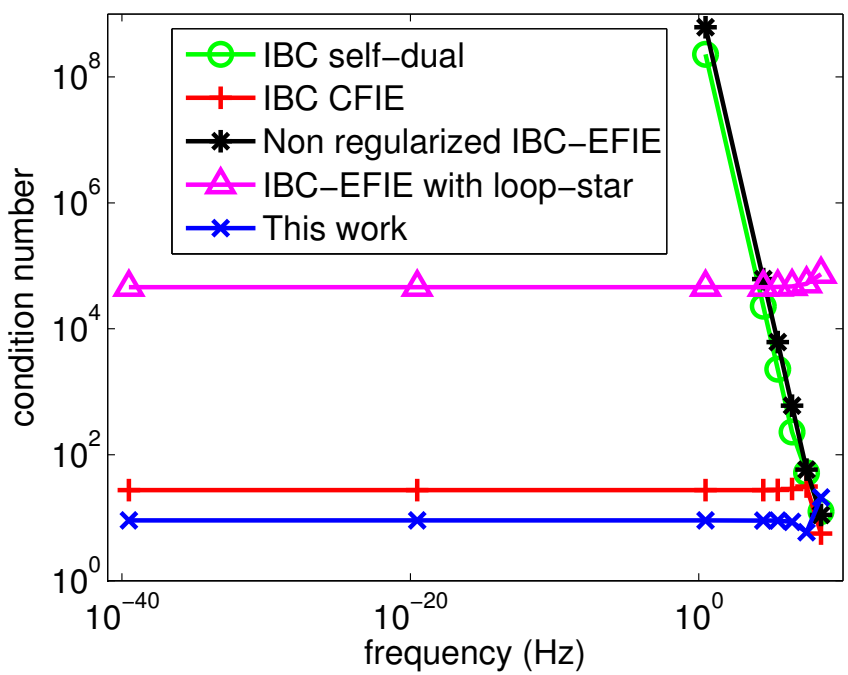

Fig. 1. Condition number as a function of the frequency on the unit sphere $(z=(0.7+0.6 i) \eta, h=0.15 \mathrm{~m})$

Again, at extremely low-frequency the scattered field of the solenoidal part of the currents should be computed using the extracted form of the Green's function in the integration $\left(\frac{e^{-i k R}-1}{4 \pi R}\right.$ instead of $\left.\frac{e^{-i k R}}{4 \pi R}\right)$ to avoid numerical cancellation.

\section{NUMERICAL RESUlTS}

A first set of tests for the new formulation has been conducted on the unit sphere, for which an analytic solution is available. Our formulation is compared with two other well-established formulations ("IBC-CFIE" refers to [4] and "IBC self-dual" refers to [6]). The "non regularized IBCEFIE" refers to the equation (8). Additionnally, to compare the behavior at low frequency, we have implemented a Loop and Star basis decomposition [16] on top of (8) with a frequency rescaling.

In Fig. 1, the condition number is plotted against the frequency on a unit sphere. The impedance is kept constant and equal to $z=(0.7+0.6 i) \eta$. The mesh parameter is set equal to $h=0.15 \mathrm{~m}$. The results clearly confirm that our scheme is immune from the low-frequency breakdown.

The behavior of the conditioning as a function of the mesh density is tested in Fig. 2 where the condition number is plotted against the average edge size on the unit sphere. The impedance and the frequency are set equal to $z=(0.7+0.6 i) \eta$ and $f=60 \mathrm{MHz}$ for all simulations. The condition number remains constant when the discretization increases showing that our equation is immune from the dense mesh breakdown while the other formulations have at least an $O\left(\frac{1}{h}\right)$ growing.

Our scheme is immune from the low-frequency breakdown also when the impedance is a function of the frequency. When the material is a conductor of conductivity $\sigma$, the surface current is assumed to flow in a boundary layer with a so called skin depth $\delta=\sqrt{\frac{2}{\sigma \mu \omega}}$. Fig.3 plots the condition number of the equation for an impedance equal to $z=1.0268 \times 10^{-7}(1+i) \sqrt{\omega}$. In this example $z=\sqrt{\frac{\mu \omega}{2 \sigma}}(1+i)$ is chosen for the copper $\left(\sigma=5.69 \times 10^{7} \mathrm{~S} / \mathrm{m}\right)$. Again, the 


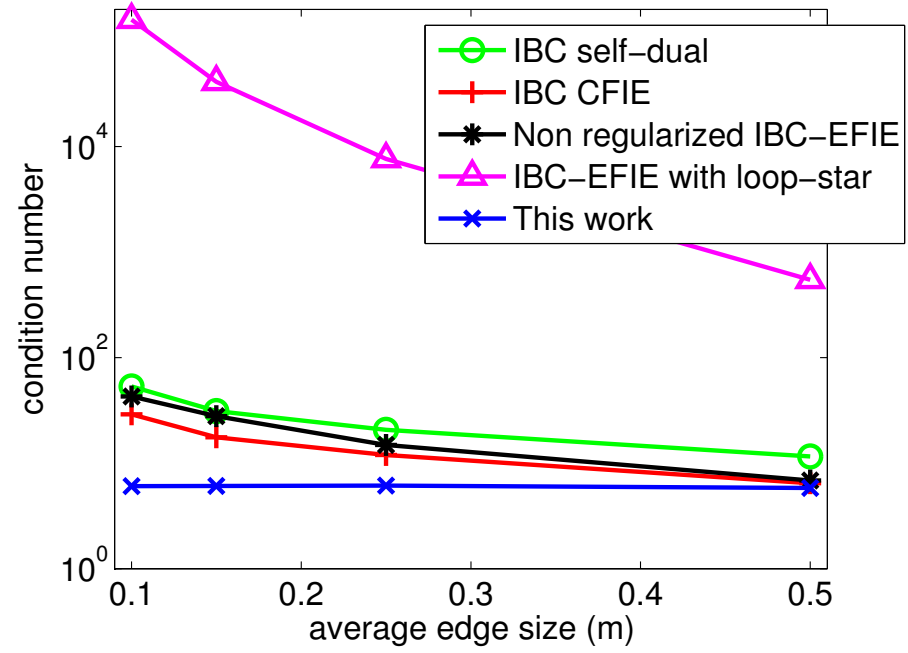

Fig. 2. Condition number as a function of the mesh size on the unit sphere mesh $(z=(0.7+0.6 i) \eta, f=60 \mathrm{MHz})$

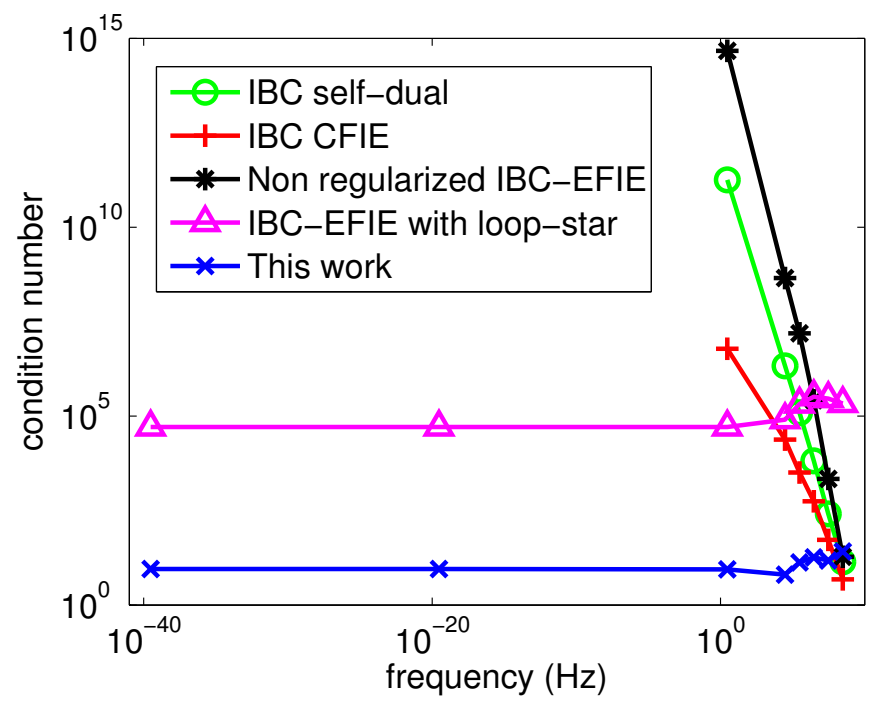

Fig. 3. Condition number as a function of the frequency on the unit sphere $\left(z=1.0268 \times 10^{-7} \sqrt{\omega}(1+i), h=0.15 \mathrm{~m}\right)$

condition number remains constant for decreasing frequencies. The reader should notice that for a wide class of more refined material models the same low frequency behaviour is recovered. In particular for the Drude model where conductivity varies as $\sigma(\omega)=\sigma_{0} /\left(1+\tau^{2} \omega^{2}\right)=\sigma_{0}+O\left(\omega^{2}\right)$. Here $\sigma_{0}$ and $\tau$ are material dependent constants.

The matching of the solution obtained with our equation with the analytic solution is verified in Fig.s 4 and 5 which compare the RCS obtained with this work and the analytic one obtained via a Mie series. The incoming plane wave is $\hat{x}^{-}$ polarized from the $+\hat{z}$ direction and the far field is computed in the $x z$ plane (VV-polarization). A matching and convergent solution is clearly evident.

The performance of the formulation on a realistic case scenarios are assessed by simulating the RCS behavior of the F-117 Nighthawk model (4593 unknowns) in Fig. 6. Fig. 7 and

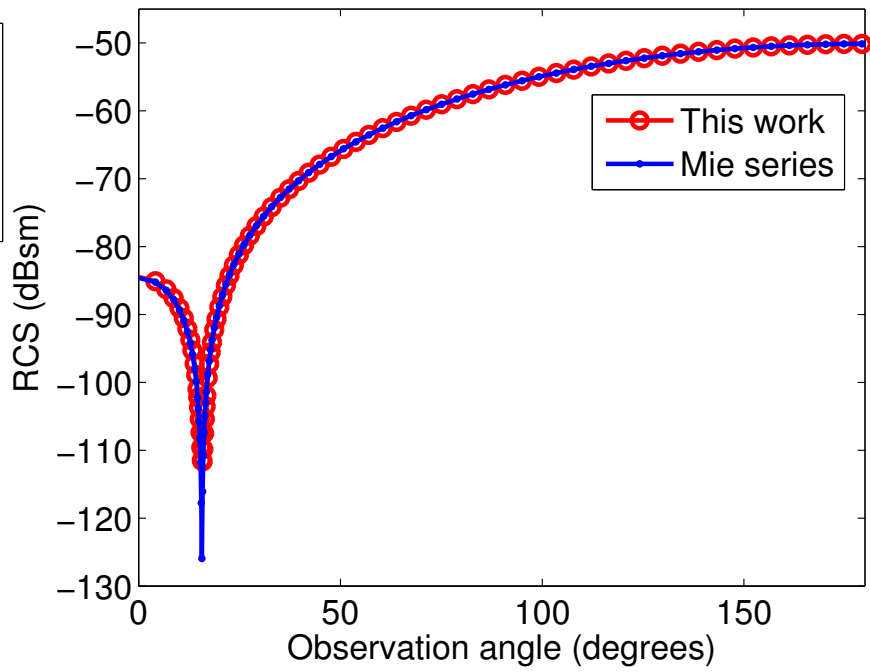

Fig. 4. RCS of a unit sphere mesh $(z=(0.8+0.6 i) \eta, f=1 \mathrm{MHz}, h=$ $0.15 \mathrm{~m}), \mathrm{VV}$-polarization

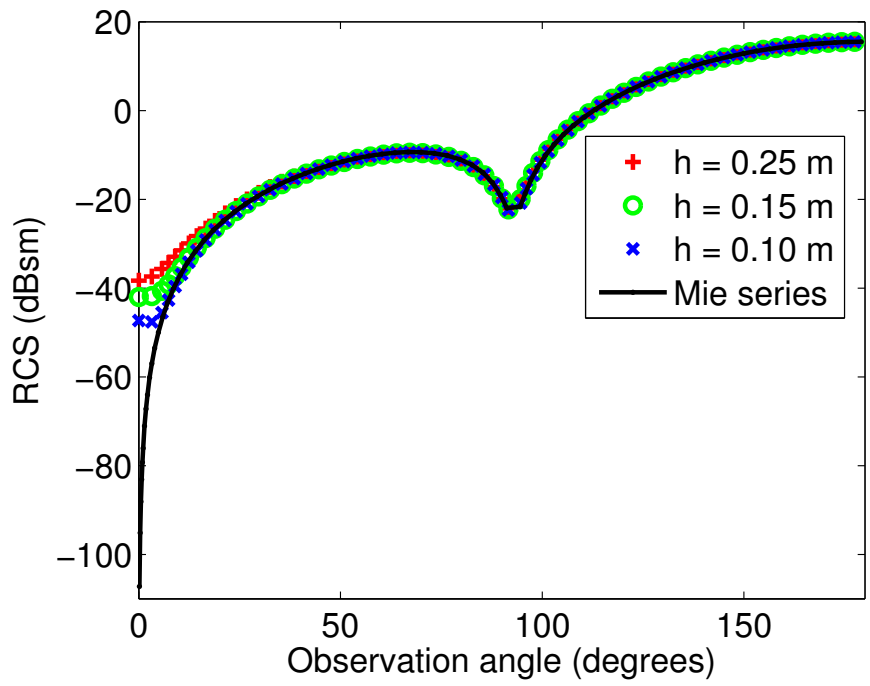

Fig. 5. RCS of a unit sphere mesh ( $z=\eta, f=100 \mathrm{MHz})$, VV-polarization

8 show the real part of the electric and the magnetic current respectively in RWG and $\mathrm{BC}$ functions basis (the incident plane wave direction is given by $\left.\varphi=20^{\circ}, \theta=110^{\circ}\right)$. Our results are compared with the IBC-CFIE [4] (the same basis are used for the solutions). For the electric current the relative error is 0.08 and for the magnetic current the relative error is 0.07 , which demonstrates that the two formulations converge to the same solution. Table I reports the partial and total timing to solve the simulation of the aircraft for different formulations. An iterative solver is used to achieve a $10^{-6}$ residual error. Although the time to build the entire matrix system is larger for our formulation, the iterative solver is much faster as it requires less iterations. This is especially relevant when the simulation requires several incident plane waves as in the following example. Fig. 9 shows the back scattering of the aircraft for several incidences as a monostatic 


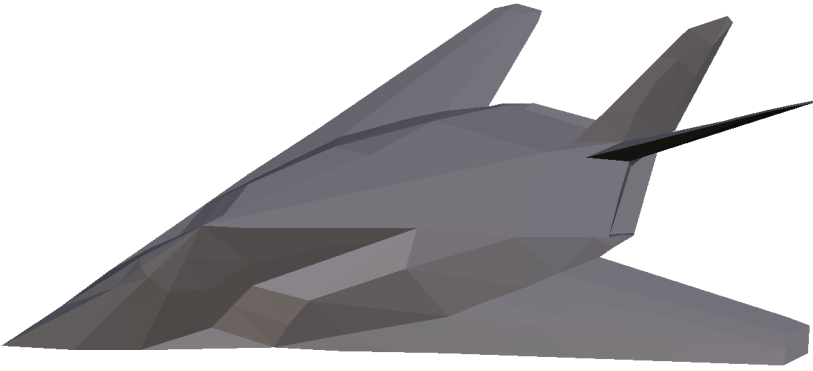

Fig. 6. F-117 Nighthawk model

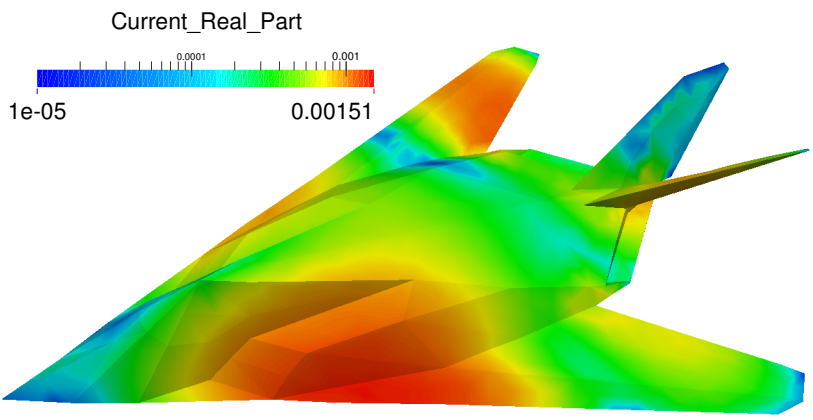

Fig. 7. Real part of the current $\mathrm{J}(z=(0.7+0.6 i) \eta, f=10 \mathrm{MHz})$

radar would measure $\left(\varphi \in[0,360]^{\circ}\right.$ and $\left.\theta=110^{\circ}\right)$. The experiment is done with and without coating $(z=\eta$ and $z=0$ respectively). The effect of the coating is clearly visible as an RCS reduction. Again the IBC-CFIE [4] is used as reference to validate the correctness of our formulation.

\section{CONCLUSION}

In this contribution, a discretization of the IBC-EFIE was introduced, together with a left-right preconditioner that results in a linear system with condition number that remains bounded

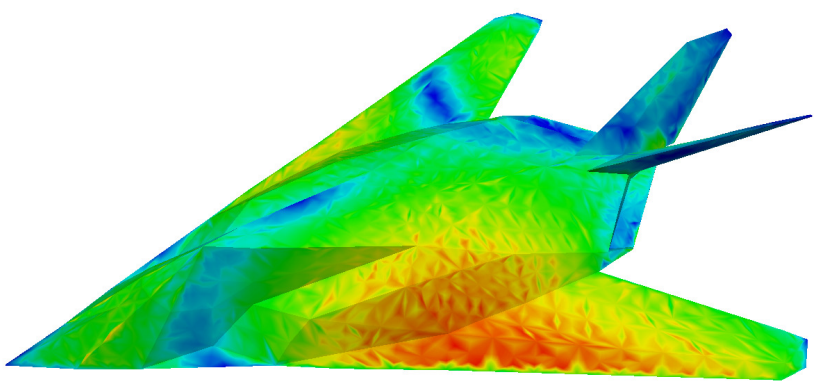

Fig. 8. Real part of the current $\mathrm{M}(z=(0.7+0.6 i) \eta, f=10 \mathrm{MHz})$
TABLE I

Partial AND total TIMING OF THE F-117 NighthaWK MODEL SIMULATION $(f=10 \mathrm{MHZ}, z=(0.7+0.6 i) \eta)$

\begin{tabular}{|c|c|c|}
\hline & This work & IBC-CFIE \\
\hline Number of iterations & 196 & 1156 \\
\hline Matrix building time (s) & 126.9 & 110.7 \\
CGS solver time (s) & 12.3 & 77.2 \\
Time per right hand side (s) & 139.2 & 187.9 \\
Total time for 100 right hand sides (s) & 1356.9 & 7830.7 \\
\hline
\end{tabular}

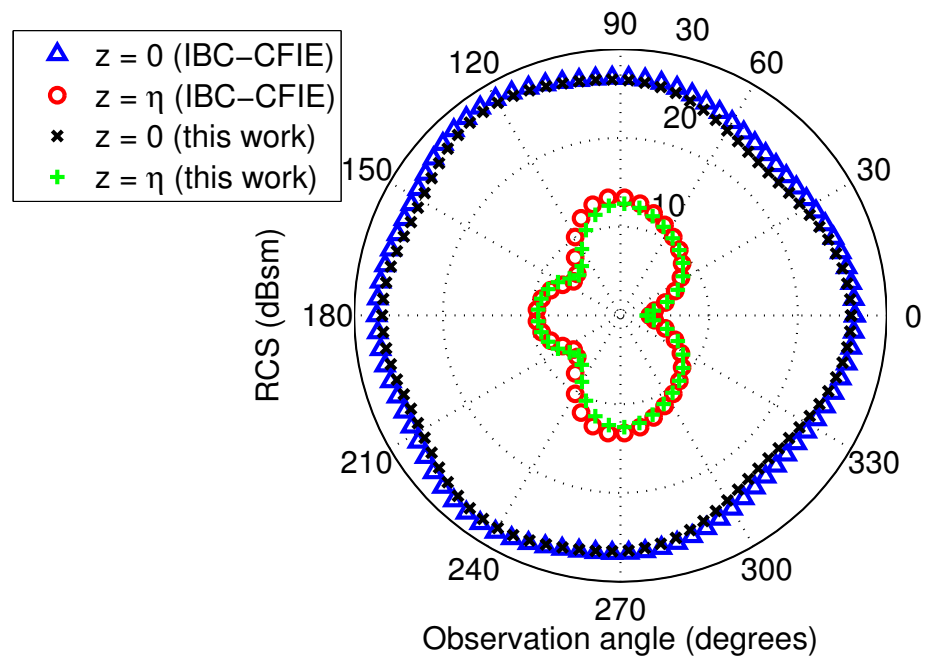

Fig. 9. RCS of the Nighthawk model at $f=10 \mathrm{MHz}$ with and without coating ( $z=\eta$ and $z=0$, respectively). The method proposed in this work is compared with the IBC-CFIE in [4].

for arbitrarily low frequencies and as the mesh density is increased. The preconditioner is purely multiplicative and is based on one hand on a Helmholtz decomposition and rescaling of the current coefficient space and on the other hand on a Calderon type preconditioner to regularize the unbounded branch in the singular value spectrum of the single layer potential. At the same time as regularizing the condition number of the system, the method guarantees that no current cancellation occurs in the solution vector and in the right hand side of the system. Numerical results demonstrate the efficiency of the proposed methodology and this both on benchmark examples and in real life scenarios.

\section{REFERENCES}

[1] A. Bendali, "Numerical analysis of the exterior boundary value problem for the time-harmonic Maxwell equations by a boundary finite element method. I. The continuous problem," Mathematics of Computation, vol. 43, no. 167, pp. 29-46, 1984.

[2] D. J. Hoppe, Impedance boundary conditions in electromagnetics. CRC Press, 1995

[3] A. W. Glisson, "Electromagnetic scattering by arbitrarily shaped surfaces with impedance boundary conditions," Radio Science, vol. 27, no. 6, pp. 935-943, Nov. 1992.

[4] P. Yla-Oijala, S. P. Kiminki, and S. Jarvenpaa, "Solving IBC-CFIE With Dual Basis Functions," IEEE Transactions on Antennas and Propagation, vol. 58, no. 12, pp. 3997-4004, Dec. 2010.

[5] W.-D. Li, W. Hong, H.-X. Zhou, and Z. Song, "Novel Buffa-Christiansen Functions for Improving CFIE With Impedance Boundary Condition," IEEE Transactions on Antennas and Propagation, vol. 60, no. 8, pp. 3763-3771, Aug. 2012. 
[6] S. Yan and J.-M. Jin, "Self-Dual Integral Equations for Electromagnetic Scattering From IBC Objects," IEEE Transactions on Antennas and Propagation, vol. 61, no. 11, pp. 5533-5546, Nov. 2013.

[7] Z. G. Qian, W. C. Chew, and R. Suaya, "Generalized Impedance Boundary Condition for Conductor Modeling in Surface Integral Equation," IEEE Transactions on Microwave Theory and Techniques, vol. 55, no. 11, pp. 2354-2364, Nov. 2007.

[8] P. Yla-Oijala, S. Kiminki, H. Wallen, and A. Sihvola, "Uniform Surface Integral Equation Formulation for Mixed Impedance Boundary Conditions," IEEE Transactions on Antennas and Propagation, vol. 63, no. 12, pp. 5718-5726, Dec. 2015

[9] F. P. Andriulli, K. Cools, H. Bagci, F. Olyslager, A. Buffa, S. Christiansen, and E. Michielssen, "A Multiplicative Calderon Preconditioner for the Electric Field Integral Equation," IEEE Transactions on Antennas and Propagation, vol. 56, no. 8, pp. 2398-2412, Aug. 2008.

[10] F. P. Andriulli, K. Cools, I. Bogaert, and E. Michielssen, "On a WellConditioned Electric Field Integral Operator for Multiply Connected Geometries," IEEE Transactions on Antennas and Propagation, vol. 61, no. 4, pp. 2077-2087, Apr. 2013.

[11] S. Rao, D. Wilton, and A. Glisson, "Electromagnetic scattering by surfaces of arbitrary shape," IEEE Transactions on Antennas and Propagation, vol. 30, no. 3, pp. 409-418, May 1982.

[12] A. Buffa and S. Christiansen, "A dual finite element complex on the barycentric refinement," Mathematics of Computation, vol. 76, no. 260, pp. 1743-1769, 2007.

[13] F. P. Andriulli, "Loop-Star and Loop-Tree Decompositions: Analysis and Efficient Algorithms," IEEE Transactions on Antennas and Propagation, vol. 60, no. 5, pp. 2347-2356, May 2012.

[14] H. Contopanagos, B. Dembart, M. Epton, J. Ottusch, V. Rokhlin, J. Visher, and S. Wandzura, "Well-conditioned boundary integral equations for three-dimensional electromagnetic scattering," IEEE Transactions on Antennas and Propagation, vol. 50, no. 12, pp. 1824-1830, Dec. 2002

[15] F. W. J. Olver and National Institute of Standards and Technology, Eds., NIST handbook of mathematical functions. Cambridge: Cambridge Univ. Press [u.a.], 2010

[16] G. Vecchi, "Loop-star decomposition of basis functions in the discretization of the EFIE," Antennas and Propagation, IEEE Transactions on, vol. 47, no. 2, pp. 339-346, 1999.

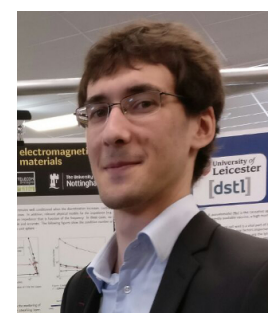

Alexandre Dély (S 16) received the M.Sc. Eng. degree from the École Nationale Supérieure des Télécommunications de Bretagne (Télécom Bretagne), France, in 2015. He is currently working toward the Ph.D. degree at the École Nationale Supérieure Mines-Télécom Atlantique (IMT Atlantique) and at The University of Nottingham. His research focusses on preconditioned and fast solution of boundary element methods and their application to impedance boundary conditions problems.

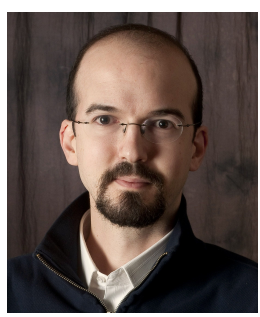

Francesco P. Andriulli (S 05, M 09, SM 11) received the Laurea in electrical engineering from the Politecnico di Torino, Italy, in 2004, the MSc in electrical engineering and computer science from the University of Illinois at Chicago in 2004, and the $\mathrm{PhD}$ in electrical engineering from the University of Michigan at Ann Arbor in 2008. From 2008 to 2010 he was a Research Associate with the Politecnico di Torino. Since 2010 he has been with the École Nationale Supérieure des Télécommunications de Bretagne (Télécom Bretagne) France, now become the École Nationale Supérieure Mines-Télécom Atlantique (IMT Atlantique), where he is currently a Full Professor. His research interests are in computational electromagnetics with focus on frequency- and time-domain integral equation solvers, well-conditioned formulations, fast solvers, low-frequency electromagnetic analysis, and simulation techniques for antennas, wireless components, microwave circuits, and biomedical applications.

Prof. Andriulli was the recipient of the best student paper award at the 2007 URSI North American Radio Science Meeting. He received the first place prize of the student paper context of the 2008 IEEE Antennas and Propagation Society International Symposium. He was the recipient of the 2009 RMTG Award for junior researchers and was awarded two URSI Young Scientist Awards at the International Symposium on Electromagnetic Theory in 2010 and 2013 where he was also awarded the second prize in the best paper contest. He also received the 2015 ICEAA IEEE-APWC Best Paper Award. In addition, he co-authored other three first prize conference papers (EMTS 2016, URSI-DE Meeting 2014, ICEAA 2009), a second prize conference paper (URSI GASS 2014), two honorable mention conference papers (ICEAA 2011, URSI/IEEEAPS 2013) and other three finalist conference papers (URSI/IEEEAPS 2012, URSI/IEEE-APS 2007, URSI/IEEE-APS 2006). Moreover, he received the 2014 IEEE AP-S Donald G. Dudley Jr. Undergraduate Teaching Award, the 2014 URSI Issac Koga Gold Medal, and the 2015 L. B. Felsen Award for Excellence in Electrodynamics.

Prof. Andriulli is a member of Eta Kappa Nu, Tau Beta Pi, Phi Kappa $\mathrm{Phi}$, and of the International Union of Radio Science (URSI). He serves as an Associate Editor for the IEEE Transactions on Antennas and Propagation, IEEE Antennas and Wireless Propagation Letters, and IEEE Access.

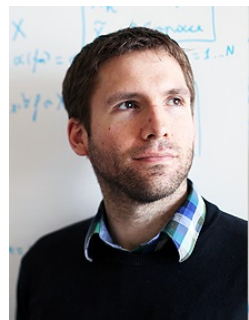

Kristof Cools received the MEng. degree in applied physics engineering and the $\mathrm{PhD}$. degree from Ghent University, Ghent, Belgium, in 2004 and 2008, respectively.

Kristof Cools is an Associate Professor in the George Green Institute for Electromagnetics Research at the University of Nottingham, Nottingham, U.K. His research interests include the spectral properties of the boundary integral operators of electromagnetics, stable and accurate discretization schemes for frequency and time domain boundary element methods, domain decomposition techniques, and on the implementations of algorithms from computational physics for high-performance computing.

Dr. Cools was the recipient of the Young Scientist Best Paper Award at the International Conference on Electromagnetics and Advanced Applications in 2008 . 